\title{
Avoiding order reduction when integrating linear initial boundary value problems with Lawson methods
}

\author{
I. Alonso-Mallo ${ }^{*}$ B. Cano ${ }^{\dagger}$ \\ IMUVA, Departamento de Matemática Aplicada, \\ Facultad de Ciencias, Universidad de Valladolid, \\ Paseo de Belén 7, 47011 Valladolid, \\ Spain \\ AND \\ N. REGUERA ${ }^{\ddagger}$ \\ IMUVA, Departamento de Matemáticas y Computación, \\ Escuela Politécnica Superior, Universidad de Burgos, \\ Avda. Cantabria, 09006 Burgos, \\ Spain
}

\begin{abstract}
Exponential Lawson methods are well known to have a severe order reduction when integrating stiff problems. In a previous paper, the precise order observed with Lawson methods when integrating linear problems is justified in terms of different conditions of annihilation on the boundary. In fact, the analysis of convergence with all exponential methods when applied to parabolic problems has always been performed under assumptions of vanishing boundary conditions for the solution. In this paper, we offer a generalization of Lawson methods in order to approximate problems with nonvanishing and even time-dependent boundary values. This technique is cheap and allows to avoid completely order reduction independently of having vanishing or non-vanishing boundary conditions.
\end{abstract}

\section{Introduction}

The advantages of using exponential methods when integrating in time partial differential equations are clear from the literature $[11,12,14]$. As they integrate the linear

\footnotetext{
*Email: isaias@mac.uva.es

${ }^{\dagger}$ Corresponding author. Email: bego@mac.uva.es

${ }^{\ddagger}$ Email: nreguera@ubu.es
} 
and stiff part of the differential equation in an exact way, methods which are explicit and linearly stable at the same time are achieved when integrating such type of problems, which is not possible with classical methods. However, up to our knowledge, exponential methods have always been applied and analysed to integrate partial differential problems under the assumption of vanishing or periodic boundary conditions. Nonvanishing boundary conditions have not been considered.

Moreover, one of the oldest particular types of exponential methods (Lawson methods [12]), which are very easy to construct from a Runge-Kutta method, have many times been excluded from the analysis [10]. (They do not even satisfy the condition of stiff order 1 for the methods considered in that paper.) In any case, a recent manuscript of the authors [4] makes a thorough study of the precise order which these methods show when integrating linear problems, which strongly depends on several conditions of annihilation on the boundary of its solution.

On the other hand, for classical methods which do have stages, such as RungeKutta, Fractional-Step-Runge-Kutta, Runge-Kutta-Nyström or Rosenbrock methods, some techniques have been suggested in the literature to avoid order reduction $[2,3]$ when integrating linear problems. These techniques are based on applying the method of lines by integrating first in time and then in space. Then, for the elliptic problems which the stages define, appropiate boundary conditions must be considered. It happens that, in the exponential case, when the problem is linear, the stages are not relevant since they are not necessary for the calculation of the numerical solution at each step. Therefore, another strategy is necessary.

In this paper, in the first place, we give a technique to deal with the problem of nonvanishing boundary conditions when integrating linear problems with Lawson methods. Moreover, we assume that the differential operator is the infinitesimal generator of a $\mathrm{C}_{0}$-semigroup and, in this way, both hyperbolic and parabolic cases are included. Secondly, we prove that the suggested technique allows to avoid order reduction completely. More precisely, the order observed is that of the underlying classical Runge-Kutta method when applied to a non-stiff problem. Besides, this technique is not expensive as it just implies to add some terms concerning boundary values which are negligible in number compared to the values to approximate in the interior of the domain. In such a way, it is not necessary to resort to methods of high stiff order (and necessarily more stages) if high accuracy is required. We also want to remark here that it is not an aim of this paper to study how to calculate in the most efficient way the necessary terms to avoid order reduction. That could be a subject of future research.

The paper is structured as follows. Section 2 gives some preliminaries. In Section 3 , the suggested technique (when integrating just in time) is described and the local and global errors without considering spatial discretization errors are analysed. In Section 4, the full discretization is considered, a framework for the analysis of the space discretization is given and the precise formulas to implement the technique after full discretization are stated. Moreover, local and global errors are again thoroughly analysed. Finally, in Section 5, some numerical experiments are given which corroborate that order reduction is avoided. Problems with vanishing and nonvanishing boundary conditions are considered as well as different time Lawson integrators and spatial 
discretizations.

\section{Preliminaries}

Let $X$ and $Y$ be two complex Banach spaces, $D(A)$ a dense subspace of $X$ and $A$ : $D(A) \subseteq X \rightarrow X, \partial: D(A) \subseteq X \rightarrow Y$ a pair of linear operators. We consider the linear abstract initial boundary value problem

$$
\begin{aligned}
u^{\prime}(t) & =A u(t)+f(t), \\
u(0) & =u_{0}, \\
\partial u(t) & =g(t),
\end{aligned}
$$

where $A$ and $\partial$ satisfy the following assumptions:

(A1) The boundary operator $\partial: D(A) \subset X \rightarrow Y$ is onto.

(A2) $\operatorname{Ker}(\partial)$ is dense in $X$ and $A_{0}: D\left(A_{0}\right)=\operatorname{ker}(\partial) \subset X \rightarrow X$, the restriction of $A$ to $\operatorname{Ker}(\partial)$, is the infinitesimal generator of a $C_{0^{-}}$semigroup $\left\{e^{t A_{0}}\right\}_{t \geq 0}$ in $X$, which type is denoted by $\omega$.

(A3) If $z \in \mathbb{C}$ satisfies $\operatorname{Re}(z)>\omega$ and $v \in Y$, then the steady state problem

$$
\begin{aligned}
& A x=z x, \\
& \partial x=v,
\end{aligned}
$$

possesses a unique solution denoted by $x=K(z) v$. Moreover, the linear operator $K(z): Y \rightarrow D(A)$ satisfies

$$
\|K(z) v\|_{X} \leq L\|v\|_{Y}
$$

where the constant $L$ holds for any $z$ such that $\operatorname{Re}(z)>\omega_{0}>\omega$.

With these assumptions, the problem (1) is well-posed in $B V / L^{\infty}$ sense [16]:

(WP1) If $u_{0} \in D(A), g:[0, T] \rightarrow Y$ is smooth enough with $\partial u_{0}=g(0)$, and $f \in$ $C^{1}([0, T], X)$, then there exists a unique solution $u \in C^{1}([0, T], X)$ of $(1)$, and

(WP2) there exist constants $M>0$ and $\alpha=\max (\omega, 0)$ such that, for $0 \leq t \leq T$,

$$
\|u(t)\|_{X} \leq M e^{\alpha t}\left(\left\|u_{0}\right\|_{X}+\|g(0)\|_{Y}+\int_{0}^{T}\left\|g^{\prime}(s)\right\|_{Y} d s+\int_{0}^{T}\|f(s)\|_{X} d s\right) .
$$

Remark 1. Instead of (A3), we can impose equivalently,

(A3') The operator $(A, \partial): D(A) \subset X \rightarrow X \times Y$ is closed.

Remark 2. Although the previous well-posedness is sufficient for our analysis, it is possible to prove the well-posedness of the problem (1) when other norms of $L^{p}$-type are used for the solution and the boundary datum [5]. 
Remark 3. From now on, we assume for the sake of simplicity that the type $\omega$ of the semigroup $\left\{e^{t A_{0}}\right\}_{t \geq 0}$ is negative. As a consequence, the semigroup decays exponentially when $t \rightarrow 0$. Moreover, the operator $A_{0}$ is invertible and, since we can take $z=0$ in (2), the operator $K(0): Y \rightarrow D(A) \subset X$ is well defined and it is continuous.

Because of hypothesis (A2), $\left\{\varphi_{j}\left(t A_{0}\right)\right\}_{j=0}^{\infty}$ are bounded operators for $t>0$, where $\left\{\varphi_{j}\right\}$ are the standard functions used in exponential methods [11], which are defined by

$$
\varphi_{j}\left(t A_{0}\right)=\frac{1}{t^{j}} \int_{0}^{t} e^{(t-\tau) A_{0}} \frac{\tau^{j-1}}{(j-1) !} d \tau,
$$

and can be calculated in a recursive way through the formula

$$
\varphi_{k+1}(z)=\frac{\varphi_{k}(z)-1 / k !}{z}, \quad z \neq 0, \quad \varphi_{k+1}(0)=\frac{1}{(k+1) !}, \quad \varphi_{0}(z)=e^{z} .
$$

These functions are well known to be bounded on the complex plane when $\operatorname{Re}(z) \leq 0$.

Since we are interested in approximations of high order, we assume that the solution of (1) is regular. We will assume that, for a natural number $p$,

$$
A^{p+1-j} u^{(j)} \in C([0, T], X), \quad 0 \leq j \leq p+1 .
$$

This assumption implies that the time derivatives of the solution are regular in space, but without to impose any restriction on the boundary values. Theorem 3.1 in [1] shows that this is satisfied when the data $u_{0}, f$ and $g$ are regular and the boundary values $\partial u_{0}, \partial f(0)$ and $g(0)$ satisfy certain natural compatibility constraints. As a consequence, from (1),

$$
u^{(j)}(t)=\sum_{l=0}^{j-1} A^{l} f^{(j-1-l)}(t)+A^{j} u(t), \quad 0 \leq j \leq p+1,
$$

which implies that the boundary values

$$
\partial A^{j} u(t)=g^{(j)}(t)-\sum_{l=0}^{j-1} \partial A^{l} f^{(j-1-l)}(t), \quad 0 \leq j \leq p+1,
$$

can be obtained from the data of the problem (1). These boundary values are crucial in our analysis below.

For the time integration, we will center on Lawson methods [12], which applied to a finite-dimensional linear problem like

$$
U^{\prime}(t)=B U(t)+F(t)
$$

where $B$ is a matrix, are described by the following formula at each step

$$
U_{n+1}=e^{k B} U_{n}+k \sum_{i=1}^{s} b_{i} e^{\left(1-c_{i}\right) k B} F\left(t_{n}+c_{i} k\right) .
$$


Here, the coefficients $\left\{b_{i}\right\},\left\{c_{i}\right\}$ are those of an underlying Runge-Kutta method. For this problem, they just correspond in fact to a quadrature rule approximation to the integral in the equality

$$
U\left(t_{n+1}\right)=e^{k B} U\left(t_{n}\right)+k \int_{0}^{1} e^{k(1-s) B} F\left(t_{n}+s k\right) d s,
$$

which is satisfied by the solutions of (9) when $t_{n+1}=t_{n}+k$. Notice that, when the method has order $p$, the corresponding quadrature rule exactly integrates all polynomials of degree $\leq p-1$. (As distinct, notice that, with exponential quadrature rules, $F$ is approximated by a polynomial and then the integral is performed exactly.)

\section{Time semidiscretization}

In this section, we give the technique to avoid in time order reduction with vanishing and non-vanishing boundary conditions. Besides, we prove the full-order convergence for the local error of the time semidiscretization.

\subsection{Description of the technique}

Our idea is to generalize the exponential Lawson method (10) to be able to use it to time integrate the initial boundary value problem (1).

When the boundary values vanish, in which case $A \equiv A_{0}$ is the infinitesimal generator of a $\mathrm{C}_{0}$-semigroup, the method (10) can be generalized in an obvious way by taking $e^{t A_{0}}$ as the semigroup generated by $A_{0}$.

The key in order to include non vanishing boundary values is to realize that $v(t)=$ $e^{t A_{0}} \alpha$ is the solution of the abstract differential problem

$$
\begin{aligned}
& v^{\prime}(t)=A_{0} v(t), \\
& v(0)=\alpha,
\end{aligned}
$$

which, with the notation of (1), corresponds to the initial boundary value problem

$$
\begin{aligned}
v^{\prime}(t) & =A v(t), \\
v(0) & =\alpha \\
\partial v(t) & =0 .
\end{aligned}
$$

Then, we add suitable boundary values to (12) and we replace $v(t)=e^{t A_{0}} \alpha$ in the exponential Lawson method with its solution. If we choose the boundary values

$$
\begin{aligned}
v^{\prime}(t) & =A v(t), \\
v(0) & =\alpha, \\
\partial v(t) & =\sum_{j=0}^{p} \frac{t^{j}}{j !} \partial A^{j} \alpha,
\end{aligned}
$$

we deduce that

$$
v(k)=\sum_{j=0}^{p} \frac{k^{j}}{j !} A^{j} \alpha+O\left(k^{p+1}\right),
$$


(see Lemma 4) and we can prove the consistency of order $p$ for the whole method just as in the case of an ordinary differential system (see Theorem 7.)

With this idea, for the time integration of (1) we suggest to advance a stepsize from $u_{n}$ in the following way

$$
u_{n+1}=\hat{u}_{n, 0}+k \sum_{i=1}^{s} b_{i} \hat{f}_{n, i}
$$

where $\hat{u}_{n, 0}$ is the value at $t=k$ of the solution of

$$
\begin{aligned}
u_{n, 0}^{\prime}(t) & =A u_{n, 0}(t) \\
u_{n, 0}(0) & =u_{n} \\
\partial u_{n, 0}(t) & =\sum_{j=0}^{p} \frac{t^{j}}{j !} \partial A^{j} u\left(t_{n}\right),
\end{aligned}
$$

and $\hat{f}_{n, i}$ is the value at $t=k\left(1-c_{i}\right)$ of the solution of

$$
\begin{aligned}
f_{n, i}^{\prime}(t) & =A f_{n, i}(t) \\
f_{n, i}(0) & =f\left(t_{n}+c_{i} k\right) \\
\partial f_{n, i}(t) & =\sum_{l=0}^{p-1} \frac{t^{l}}{l !} \partial A^{l} f\left(t_{n}+c_{i} k\right) .
\end{aligned}
$$

We notice that the boundary values in (14) can always be calculated in terms of data taking (8) into account.

\subsection{Local error of the time semidiscretization}

In order to study the local error, we consider the value obtained in (13) starting from $u\left(t_{n}\right)$ in (14), and (15). Then, we obtain

$$
\bar{u}_{n+1}=\hat{\bar{u}}_{n, 0}+k \sum_{i=1}^{s} b_{i} \hat{f}_{n, i},
$$

where $\hat{\bar{u}}_{n, 0}$ is the value at $t=k$ of the solution of

$$
\begin{aligned}
\bar{u}_{n, 0}^{\prime}(t) & =A \bar{u}_{n, 0}(t) \\
\bar{u}_{n, 0}(0) & =u\left(t_{n}\right), \\
\partial \bar{u}_{n, 0}(t) & =\partial\left(\sum_{j=0}^{p} \frac{t^{j}}{j !} A^{j} u\left(t_{n}\right)\right)
\end{aligned}
$$

and $\hat{f}_{n, i}$ is that defined in $(15)$.

To bound the local error $\rho_{n+1}=\bar{u}_{n+1}-u\left(t_{n+1}\right)$, we use the following lemma

Lemma 4. Let us assume hypotheses (A1)-(A2)-(A3) of Section 2, and that $\alpha \in D(A)$, $\beta \in D\left(A^{p+1}\right)$ satisfy $\partial \alpha=\partial \beta$. Consider the auxiliary problem

$$
\begin{aligned}
v^{\prime}(t) & =A v(t), \\
v(0) & =\alpha, \\
\partial v(t) & =\partial z(t),
\end{aligned}
$$


where

$$
z(t)=\sum_{j=0}^{p} \frac{t^{j}}{j !} A^{j} \beta
$$

Then,

$$
v(t)=e^{t A_{0}}(\alpha-\beta)+\sum_{j=0}^{p} \frac{t^{j}}{j !} A^{j} \beta+t^{p+1} \varphi_{p+1}\left(t A_{0}\right) A^{p+1} \beta
$$

and

$$
A v(t)=e^{t A_{0}} A_{0}(\alpha-\beta)+\sum_{j=0}^{p-1} \frac{t^{j}}{j !} A^{j+1} \beta+t^{p} \varphi_{p}\left(t A_{0}\right) A^{p+1} \beta,
$$

where $\varphi_{p}(z)$ and $\varphi_{p+1}(z)$ are defined in (4).

Proof. By considering $w(t)=v(t)-z(t)$ one gets

$$
w^{\prime}(t)=A_{0} w(t)+\frac{t^{p}}{p !} A^{p+1} \beta, \quad w(0)=\alpha-\beta,
$$

which means that

$$
w(t)=e^{t A_{0}}(\alpha-\beta)+\int_{0}^{t} e^{(t-\tau) A_{0}} \frac{\tau^{p}}{p !} A^{p+1} \beta d \tau,
$$

and (18) follows. Now, we can apply Lemma 1 in [4] (see also [17]) and we deduce that

$$
\int_{0}^{t} e^{(t-\tau) A_{0}} \frac{\tau^{p}}{p !} A^{p+1} \beta d \tau \in D\left(A_{0}\right)
$$

and

$$
A_{0} \int_{0}^{t} e^{(t-\tau) A_{0}} \frac{\tau^{p}}{p !} A^{p+1} \beta d \tau=-\frac{t^{p}}{p !} A^{p+1} \beta+\int_{0}^{t} e^{(t-\tau) A_{0}} \frac{\tau^{p-1}}{(p-1) !} A^{p+1} \beta d \tau,
$$

which proves the second formula.

From this result, we can study more thoroughly $\bar{u}_{n, 0}(t)$ and $f_{n, i}(t)$.

Lemma 5. Let us assume hypotheses (A1)-(A2)-(A3)-(WP1)-(WP2) of Section 2. Then,

$$
\bar{u}_{n, 0}(t)=\sum_{j=0}^{p} \frac{t^{j}}{j !} A^{j} u\left(t_{n}\right)+t^{p+1} \varphi_{p+1}\left(t A_{0}\right) A^{p+1} u\left(t_{n}\right)
$$

and

$$
A \bar{u}_{n, 0}(t)=\sum_{j=0}^{p-1} \frac{t^{j}}{j !} A^{j+1} u\left(t_{n}\right)+t^{p} \varphi_{p}\left(t A_{0}\right) A^{p+1} u\left(t_{n}\right) .
$$


Lemma 6. Let us assume hypotheses (A1)-(A2)-(A3)-(WP1)-(WP2) of Section 2. Then,

$$
f_{n, i}(t)=\sum_{j=0}^{p-1} \frac{t^{j}}{j !} A^{j} f\left(t_{n}+c_{i} k\right)+t^{p} \varphi_{p}\left(t A_{0}\right) A^{p} f\left(t_{n}+c_{i} k\right)
$$

and

$$
A f_{n, i}(t)=\sum_{j=0}^{p-2} \frac{t^{j}}{j !} A^{j+1} f\left(t_{n}+c_{i} k\right)+t^{p-1} \varphi_{p-1}\left(t A_{0}\right) A^{p} f\left(t_{n}+c_{i} k\right) .
$$

We now deduce the consistency of order $p$.

Theorem 7. Under hypotheses (A1)-(A2)-(A3)-(WP1)-(WP2) of Section 2, and assuming that $u(t) \in D\left(A^{p+1}\right)$ for $t \in[0, T]$, with $A^{j} u \in C([0, T], X), j=0, \ldots, p+1$, and $f^{(l)}(t) \in D\left(A^{j}\right)$ for $t \in[0, T]$, with $A^{j} f^{(l)}(t) \in C([0, T], X), l=0, \ldots, p+1-j$, $j=1, \ldots, p$, whenever the method has order $p$, the local error satisfies

$$
\rho_{n+1} \equiv \bar{u}_{n+1}-u\left(t_{n+1}\right)=O\left(k^{p+1}\right) .
$$

where the constant in Landau notation for the residue depends on a bound for $A^{p+1} u$, $A^{p} f, A^{j} f^{(p-j)}, j=0, \ldots, p-1$, and the bound for the operators $\varphi_{p+1}\left(k A_{0}\right)$ and $\varphi_{p}((1-$ $\left.\left.c_{i}\right) k A_{0}\right)$.

Proof. By considering $t=k$ in Lemma 5 and $t=\left(1-c_{i}\right) k$ in Lemma 6 , it is clear that

$$
\bar{u}_{n+1}=\sum_{j=0}^{p} \frac{k^{j}}{j !} A^{j} u\left(t_{n}\right)+k \sum_{i=1}^{s} b_{i} \sum_{j=0}^{p-1} \frac{k^{j}\left(1-c_{i}\right)^{j}}{j !} A^{j} f\left(t_{n}+c_{i} k\right)+O\left(k^{p+1}\right) .
$$

where the residue depends on $A^{p+1} u, A^{p} f$ and the bound for the operators $\varphi_{p+1}\left(k A_{0}\right)$ and $\varphi_{p}\left(\left(1-c_{i}\right) k A_{0}\right)$. This can be rewritten as

$$
\begin{aligned}
\bar{u}_{n+1}= & u\left(t_{n}\right)+\sum_{j=1}^{p} \frac{k^{j}}{j !}\left[A^{j} u\left(t_{n}\right)+j \sum_{i=1}^{s} b_{i}\left(1-c_{i}\right)^{j-1} \sum_{l=0}^{p-j} \frac{c_{i}^{l} k^{l}}{l !} A^{j-1} f^{(l)}\left(t_{n}\right)\right]+O\left(k^{p+1}\right) \\
= & u\left(t_{n}\right)+\sum_{\tilde{\jmath}=1}^{p} \frac{k^{\tilde{\jmath}}}{\tilde{\mathrm{j}} !}\left[A^{\tilde{\mathrm{j}}} u\left(t_{n}\right)+\sum_{l=0}^{\tilde{\mathrm{j}}-1} \frac{\tilde{\mathrm{j}}(\tilde{\mathrm{j}}-1) \ldots(\tilde{\mathrm{j}}-l)}{l !}\left(\sum_{i=1}^{s} b_{i}\left(1-c_{i}\right)^{\tilde{\mathrm{j}}-l-1} c_{i}^{l}\right) A^{\tilde{\mathrm{j}}-l-1} f^{(l)}\left(t_{n}\right)\right] \\
& +O\left(k^{p+1}\right),
\end{aligned}
$$

where the change of variables $\tilde{j}=j+l$ has been used for the second term in the bracket of (19), and the residue now also depends on a bound for $A^{j} f^{(p-j)}, j=0, \ldots, p-1$. Now, it suffices to take into account that the quadrature rule which is associated to the method integrates exactly polynomials of degree $\leq p-1$. Then, for $\tilde{\mathrm{j}} \leq p$,

$$
\sum_{i=1}^{s} b_{i}\left(1-c_{i}\right)^{\tilde{\jmath}-l-1} c_{i}^{l}=\int_{0}^{1}(1-x)^{\tilde{\jmath}-l-1} x^{l} d x=\frac{l !}{\tilde{\jmath}(\tilde{\jmath}-1) \ldots(\tilde{\jmath}-l)},
$$


where integration-by-parts has been used for the second equality. This implies, from (19) and (7), that

$$
\begin{aligned}
\bar{u}_{n+1} & =u\left(t_{n}\right)+\sum_{\tilde{\jmath}=1}^{p} \frac{k^{\tilde{\jmath}}}{\tilde{\jmath} !}\left[A^{\tilde{\jmath}} u\left(t_{n}\right)+\sum_{l=0}^{\tilde{\jmath}-1} A^{\tilde{\jmath}-l-1} f^{(l)}\left(t_{n}\right)\right]+O\left(k^{p+1}\right) \\
& =u\left(t_{n}\right)+\sum_{\tilde{\jmath}=1}^{p} \frac{k^{\tilde{\jmath}}}{\tilde{\jmath} !} u^{(\tilde{\jmath})}\left(t_{n}\right)+O\left(k^{p+1}\right)=u\left(t_{n+1}\right)+O\left(k^{p+1}\right),
\end{aligned}
$$

which proves the theorem.

\section{Full discretization}

In this section, we study the full discretization of problem (1). A crucial point is to take into account that the boundary values are nonvanishing.

When problem (1) has homogeneous boundary conditions, it can be written as the initial value problem

$$
\begin{aligned}
& u^{\prime}(t)=A_{0} u(t)+f(t), \\
& u(0)=u_{0},
\end{aligned}
$$

where, in our more general approach, the operator $A_{0}: D\left(A_{0}\right) \subset X \rightarrow X$ is the generator of a $\mathrm{C}_{0}$-semigroup. To reduce a problem with nonvanishing boundary conditions to one of that type, we need to assume that it is possible to find a suitable extension of the boundary value data to the whole domain where the solution is defined. That is, for each $t \in[0, T]$, the boundary value $g(t)$ is extended to an element $x_{g}(t) \in D(A)$ such that $\partial x_{g}(t)=g(t)$. Then, the function $v(t)=u(t)-x_{g}(t)$ satisfies the initial boundary value problem

$$
\begin{aligned}
v^{\prime}(t) & =u^{\prime}(t)-x_{g}^{\prime}(t)=A u(t)+f(t)-x_{g}^{\prime}(t)=A v(t)+f(t)-x_{g}^{\prime}(t)+A x_{g}(t), \\
v(0) & =u(0)-x_{g}(0), \\
\partial v(t) & =\partial u(t)-\partial x_{g}(t)=0,
\end{aligned}
$$

which can be written as (20).

However, this is not a practical way to obtain a numerical approximation since, at least, two problems come up when this idea is carried out. Firstly, the extension operator is not easily obtained. A possibility is to consider the extension operator $x_{g}(t)=K(0) g(t)$ in $(3)$, but it is not easy at all to obtain it for multidimensional problems. Moreover, approximating it in a numerical way at each step would lead to the necessity of solving a linear system at each step and to numerically calculate $x_{g}^{\prime}\left(t_{n}\right)$. Secondly, it is necessary to choose carefully $x_{g}(t)$ in order to avoid completely the order reduction phenomenon. For example, the choice $x_{g}(t)=K(0) g(t)$ only permits, in general, to recover an order of consistency and convergence. If we want to avoid completely the order reduction, the extension operator must be obtained in a more complex way [7]. 
As a suitable alternative, we use a spatial discretization which takes into account the boundary values in a natural way (cf. with $[1,2]$ ).

\subsection{Spatial discretization}

We first consider an abstract spatial discretization. As we will check in the examples of Section 5, this framework includes problems which are discretized in space by usual Galerkin finite element and finite difference techniques.

Let us denote by $h \in\left(0, h_{0}\right]$ the parameter of the spatial discretization. Let $X_{h}$ be a family of finite dimensional spaces, approximating $X$. The norm in $X_{h}$ is denoted by $\|\cdot\|_{h}$. We suppose that

$$
X_{h}=X_{h, 0} \oplus X_{h, b}
$$

in such a way that the internal approximation is collected in $X_{h, 0}$ and $X_{h, b}$ accounts for the boundary values.

The elements in $D\left(A_{0}\right)$, which are regular in space and have vanishing boundary conditions, can be approximated in $X_{h, 0}$. However, it is possible to consider elements $u \in X$ which are regular in space but with non-vanishing boundary conditions, i.e. $u \in D(A)$. Then, it is necessary to use the whole discrete space $X_{h}$.

Since the solution is known at the boundary, our goal is to obtain a value in $X_{h, 0}$ which is a good approximation inside the domain.

Let us take a projection operator

$$
L_{h}: X \rightarrow X_{h, 0}
$$

When $x \in D\left(A_{0}\right), L_{h} x$ will be its best approximation in $X_{h, 0}$. We also assume that there exists another operator

$$
Q_{h}: Y \rightarrow X_{h, b}
$$

which permits to discretize spatially the boundary values.

Then, we define

$$
P_{h}:=\left(L_{h}-L_{h} Q_{h} \partial\right): D(A) \rightarrow X_{h, 0}
$$

which is the internal spatial approximation of an element in $D(A)$.

On the other hand, the operator $A: D(A) \subset X \rightarrow X$ is approximated by means of the operators

$$
A_{h}: X_{h} \rightarrow X_{h, 0}
$$

in such a way that $A_{h, 0}: X_{h, 0} \rightarrow X_{h, 0}$, the restrictions of $A_{h}$ to the subspaces $X_{h, 0}$, are approximations of $A_{0}: D\left(A_{0}\right) \subset D(A) \rightarrow X$. Therefore, when $x_{h}=x_{h, 0}+x_{h, b} \in$ $X_{h, 0} \oplus X_{h, b}=X_{h}$, we have

$$
A_{h} x_{h}=A_{h, 0} x_{h, 0}+A_{h} x_{h, b}
$$


We pose the following semidiscrete problem: Find $U_{h}(t) \in X_{h, 0}$ such that

$$
\begin{aligned}
U_{h}^{\prime}(t)+L_{h} Q_{h} g^{\prime}(t) & =A_{h}\left(U_{h}(t)+Q_{h} g(t)\right)+L_{h} f(t), \\
U_{h}(0)+L_{h} Q_{h} g(0) & =L_{h} u(0),
\end{aligned}
$$

or, equivalently,

$$
\begin{aligned}
U_{h}^{\prime}(t) & =A_{h, 0} U_{h}(t)+A_{h} Q_{h} g(t)+L_{h} Q_{h}\left(\partial f(t)-g^{\prime}(t)\right)+P_{h} f(t), \\
U_{h}(0) & =P_{h} u(0),
\end{aligned}
$$

which results from the discretization in space of problem (1).

The subsequent analysis is carried out under the following hypotheses, which are very close to those in [6] (see also [1]).

(H1) The operators $A_{h, 0}$ are invertible and generate uniformly bounded $C_{0}$-semigroups $e^{t A_{h, 0}}$ on $X_{h, 0}$ satisfying

$$
\left\|e^{t A_{h, 0}}\right\|_{h} \leq M
$$

where $M \geq 1$ is a constant.

(H2) For each $u \in X$,

$$
\left\|L_{h} u\right\|_{h} \leq C\|u\|_{X}
$$

where $C$ is constant, and, for each $v \in Y$,

$$
\left\|Q_{h} v\right\|_{h} \leq \gamma_{h}\|v\|_{Y}
$$

where $\gamma_{h}$ may increase in a moderate way when $h \rightarrow 0$.

(H3) We define the elliptic projection $R_{h}: D(A) \rightarrow X_{h, 0}$ as follows. If $u \in D(A)$, then $R_{h} u$ satisfies

$$
A_{h}\left(R_{h} u+Q_{h} \partial u\right)=L_{h} A u
$$

or, equivalently,

$$
R_{h} u=A_{h, 0}^{-1}\left(L_{h} A u-A_{h} Q_{h} \partial u\right) .
$$

Notice that the elliptic projection $R_{h} u$ is the discretized solution of the steady state problem with exact solution $u$.

We assume that there exists a subspace $Z$ of $X$, such that, for $u \in Z$,

(a) $A_{0}^{-1} u \in Z$ and $e^{t A_{0}} u \in Z$, for $t \geq 0$,

(b) for some $\varepsilon_{h}$ which is small with $h$,

$$
\left\|\left(L_{h}-L_{h} Q_{h} \partial\right) u-R_{h} u\right\|_{h}=\left\|P_{h} u-R_{h} u\right\|_{h} \leq \varepsilon_{h}\|u\|_{Z},
$$

That is, the solution of the spatial discretization of a steady state problem with solution $u$ is a good approximation of $u \in Z$. 


\subsection{Time integration. Lawson methods.}

We now obtain a full discretization of (1). Notice that, since (21) is in practice an ordinary differential system, it is possible to obtain a full discretization by applying a standard Lawson method to it. However, this method comes to a very inaccurate result due to the high order reduction phenomenon arising when nonvanishing boundary values are present. (When $g \neq 0$, the source term in (21) is, in practice, infinitely large when $h$ goes to zero.)

Our idea is to begin with the time semidiscretization (13) by using the solutions of problems (14) and (15). Then, we apply the space discretization described above to those problems and we obtain the scheme

$$
U_{h, n+1}=\hat{U}_{h, n, 0}+k \sum_{j=1}^{s} b_{j} \hat{F}_{h, n, j}
$$

where $\hat{U}_{h, n, 0}$ equals $U_{h, n, 0}(k)$ with $U_{h, n, 0}(t) \in X_{h, 0}$ the solution of

$$
\begin{aligned}
U_{h, n, 0}^{\prime}(t)+L_{h} Q_{h} \partial u_{n, 0}^{\prime}(t) & =A_{h}\left(U_{h, n, 0}(t)+Q_{h} \partial u_{n, 0}(t)\right) \\
U_{h, n, 0}(0) & =U_{h, n}
\end{aligned}
$$

and $u_{n, 0}$ is the same as in (14) and, for $i=1, \ldots, s, \hat{F}_{h, n, i}=F_{h, n, i}\left(\left(1-c_{i}\right) k\right)$ with $F_{h, n, i}(t) \in X_{h, 0}$ the solution of

$$
\begin{aligned}
F_{h, n, i}^{\prime}(t)+L_{h} Q_{h} \partial f_{n, i}^{\prime}(t) & =A_{h}\left(F_{h, n, i}(t)+Q_{h} \partial f_{n, i}(t)\right), \\
F_{h, n, i}(0)+L_{h} Q_{h} \partial f\left(t_{n}+c_{i} k\right) & =L_{h} f\left(t_{n}+c_{i} k\right),
\end{aligned}
$$

where $f_{n, i}$ is the same as in (15). Moreover, we will assume that we take, as initial condition,

$$
U_{h, 0}=P_{h} u(0)
$$

\subsubsection{Final formula for the implementation}

In this section, we state the final formula to be implemented in order to integrate the boundary value problem with possibly nonhomogeneous boundary conditions and avoiding order reduction. We will state how to obtain the exact solution of (28) and (29) through the functions $\left\{\varphi_{j}\right\}$. 
Theorem 8. The numerical solution in (27) can be written as

$$
\begin{aligned}
U_{h, n+1}= & e^{k A_{h, 0}} U_{h, n}+\sum_{j=1}^{p} k^{j} \varphi_{j}\left(k A_{h, 0}\right)\left[A_{h} Q_{h} \partial A^{j-1} u\left(t_{n}\right)-L_{h} Q_{h} \partial A^{j} u\left(t_{n}\right)\right] \\
& +k^{p+1} \varphi_{p+1}\left(k A_{h, 0}\right) A_{h} Q_{h} \partial A^{p} u\left(t_{n}\right) \\
& + \\
+ & \sum_{i=1}^{s} b_{i}\left[e^{\left(1-c_{i}\right) k A_{h, 0}} P_{h} f\left(t_{n}+c_{i} k\right)\right. \\
& +\sum_{l=1}^{p-1}\left(1-c_{i}\right)^{l} k^{l} \varphi_{l}\left(\left(1-c_{i}\right) k A_{h, 0}\right)\left[A_{h} Q_{h} \partial A^{l-1} f\left(t_{n}+c_{i} k\right)-L_{h} Q_{h} \partial A^{l} f\left(t_{n}+c_{i} k\right)\right] \\
& \left.+\left(1-c_{i}\right)^{p} k^{p} \varphi_{p}\left(\left(1-c_{i}\right) k A_{h, 0}\right) A_{h} Q_{h} \partial A^{p-1} f\left(t_{n}+c_{i} k\right)\right],
\end{aligned}
$$

where $u\left(t_{n}\right)$ is the solution of (1) we want to approximate and $\partial A^{j} u\left(t_{n}\right)$ is calculated in terms of the data $g$ and $f$ of (1) through (8).

Proof. Integrating exactly (28) and (29), using the boundary values in (14)-(15) and the definition of the functions $\left\{\varphi_{j}\right\}(4)$, the method can be written as

$$
\begin{aligned}
& U_{h, n+1}= e^{k A_{h, 0}} U_{h, n}+\int_{0}^{k} e^{(k-s) A_{h, 0}}\left[A_{h} Q_{h} \partial u_{n, 0}(s)-L_{h} Q_{h} \partial u_{n, 0}^{\prime}(s)\right] d s \\
&+k \sum_{i=1}^{s} b_{i}\left[e^{\left(1-c_{i}\right) k A_{h, 0}} P_{h} f\left(t_{n}+c_{i} k\right)\right. \\
&\left.+\int_{0}^{\left(1-c_{i}\right) k} e^{(k-s) A_{h, 0}}\left[A_{h} Q_{h} \partial f_{n, i}(s)-L_{h} Q_{h} \partial f_{n, i}^{\prime}(s)\right] d s\right] \\
&= e^{k A_{h, 0}} U_{h, n}+\sum_{j=0}^{p} k^{j+1} \varphi_{j+1}\left(k A_{h, 0}\right) A_{h} Q_{h} \partial A^{j} u\left(t_{n}\right) \\
&-\sum_{j=1}^{p} k^{j} \varphi_{j}\left(k A_{h, 0}\right) L_{h} Q_{h} \partial A^{j} u\left(t_{n}\right) \\
&+k \sum_{i=1}^{s} b_{i}\left[e^{\left(1-c_{i}\right) k A_{h, 0}} P_{h} f\left(t_{n}+c_{i} k\right)\right. \\
& \quad+\sum_{l=0}^{p-1}\left(1-c_{i}\right)^{l+1} k^{l+1} \varphi_{l+1}\left(\left(1-c_{i}\right) k A_{h, 0}\right) A_{h} Q_{h} \partial A^{l} f\left(t_{n}+c_{i} k\right) \\
&\left.\quad-\sum_{l=1}^{p-1}\left(1-c_{i}\right)^{l} k^{l} \varphi_{l}\left(\left(1-c_{i}\right) k A_{h, 0}\right) L_{h} Q_{h} \partial A^{l} f\left(t_{n}+c_{i} k\right)\right]
\end{aligned}
$$

from what the result follows.

Remark 9. In [4], it is well justified that no order reduction turns up with the standard Lawson method (without avoiding order reduction) when there is enough regularity in 
space and the following vanishing boundary conditions are satisfied

$$
\partial u(t)=\partial A u(t)=\ldots=\partial A^{p} u(t)=0 .
$$

This is in correspondance with the fact that the standard Lawson method in such a case is like (31) but eliminating all terms which contain the functions $\left\{\varphi_{j}\right\}$. This comes from the fact that, applying the space discretization to (1) in such a case and considering (21), the following problem arises

$$
U_{h}^{\prime}(t)=A_{h, 0} U_{h}(t)+P_{h} f(t) .
$$

Time integration with (10) explains the remark.

Remark 10. We remark that, for many space discretizations, for $v \in Y, A_{h} Q_{h} v$ and $L_{h} Q_{h} v$ are local in the sense that they vanish in the interior of the domain $\Omega$ (or great part of it). In such a way, the calculation of the terms which contain the functions $\left\{\varphi_{j}\right\}$ is much cheaper than it could be expected at first sight. Just some columns of the matrices which represent $\varphi_{j}\left(s k A_{h, 0}\right)$ are necessary. That is thoroughly explained with an example in Subsection 5.3.1.

\subsubsection{Local errors}

In order to define the local error, we consider

$$
\bar{U}_{h, n+1}=\hat{\bar{U}}_{h, n, 0}+k \sum_{j=1}^{s} b_{j} \hat{\bar{F}}_{h, n, j},
$$

where $\hat{\bar{U}}_{h, n, 0}$ equals $\bar{U}_{h, n, 0}(k)$, with $\bar{U}_{h, n, 0}(t)$ the solution of

$$
\begin{aligned}
\bar{U}_{h, n, 0}^{\prime}(t)+L_{h} Q_{h} \partial \bar{u}_{n, 0}^{\prime}(t) & =A_{h}\left(\bar{U}_{h, n, 0}(t)+Q_{h} \partial \bar{u}_{n, 0}(t)\right), \\
\bar{U}_{h, n, 0}(0) & =R_{h} u\left(t_{n}\right),
\end{aligned}
$$

and, for $j=1, \ldots, s, \hat{\bar{F}}_{h, n, j}=\bar{F}_{h, n, j}\left(\left(1-c_{j}\right) k\right)$ with $\bar{F}_{h, n, j}(t)$ the solution of

$$
\begin{aligned}
\bar{F}_{h, n, j}^{\prime}(t)+L_{h} Q_{h} \partial f_{n, j}^{\prime}(t) & =A_{h}\left(\bar{F}_{h, n, j}(t)+Q_{h} \partial f_{n, j}(t)\right), \\
\bar{F}_{h, n, j}(0) & =R_{h} f\left(t_{n}+c_{j} k\right) .
\end{aligned}
$$

We now define the local error at $t=t_{n}$ as

$$
\rho_{h, n}=R_{h} u\left(t_{n}\right)-\bar{U}_{h, n},
$$

and study its behaviour in the following theorem.

Theorem 11. Under the assumptions of Section 2, if $u$ and $f$ in (1) satisfy

$$
A^{j} u(t) \in Z, \quad j=0, \ldots, p+1, \quad A^{j} f(t) \in Z, \quad j=0, \ldots, p, \quad t \in[0, T],
$$


for the space $Z$ which is introduced in (H3) and the method has order $p$,

$$
\rho_{h, n+1}=O\left(\varepsilon_{h} k^{p+1}+k^{p+1}+\gamma_{h} k^{p+1}\right)+O\left(k \varepsilon_{h}\right)=O\left(\gamma_{h} k^{p+1}+k \varepsilon_{h}\right),
$$

where $\varepsilon_{h}$ and $\gamma_{h}$ and are those in (24)-(26) and the constants in Landau notation are independent of $k$ and $h$. (In fact, they do depend on bounds for $A^{j} u, j=1, \ldots, p+1$, $A^{j} f, j=1, \ldots, p$ and $\varphi_{j}\left(s A_{0}\right), j=p-1, p, p+1$.)

Proof. From the definition of $\rho_{h, n}$,

$$
\begin{aligned}
\rho_{h, n+1} & =R_{h} u\left(t_{n+1}\right)-\bar{U}_{h, n+1} \\
& =\left(R_{h} u\left(t_{n+1}\right)-R_{h} \bar{u}_{n+1}\right)+\left(R_{h} \bar{u}_{n+1}-\bar{U}_{h, n+1}\right) .
\end{aligned}
$$

Notice that, using (34), Lemmas 5 and 6, hypothesis (a) in (H3), and the recursive definition of the functions $\varphi_{j}(z)(5)$, we deduce that $\rho_{n+1} \in Z$. Then, for the first term in parenthesis and using Theorem 7, (23)-(24) and (26),

$$
\begin{aligned}
R_{h} u\left(t_{n+1}\right)-R_{h} \bar{u}_{n+1} & =R_{h} \rho_{n+1} \\
& =\left(R_{h}-P_{h}\right) \rho_{n+1}+L_{h} \rho_{n+1}-L_{h} Q_{h} \partial \rho_{n+1} \\
& =O\left(\varepsilon_{h} k^{p+1}+k^{p+1}+\gamma_{h} k^{p+1}\right),
\end{aligned}
$$

Moreover, the implicit constant in Landau notation is independent of $k$ and $h$. Now, we apply the operator $R_{h}$ to (16) and use (25),

$$
\begin{aligned}
R_{h} \bar{u}_{n, 0}^{\prime}(t)+L_{h} Q_{h} \partial \bar{u}_{n, 0}^{\prime}(t) & =R_{h} A \bar{u}_{n, 0}(t)+L_{h} Q_{h} \partial \bar{u}_{n, 0}^{\prime}(t) \\
& =L_{h} A \bar{u}_{n, 0}(t)+\left(R_{h}-L_{h}\right) A \bar{u}_{n, 0}(t)+L_{h} Q_{h} \partial A \bar{u}_{n, 0}(t) \\
& =A_{h}\left(R_{h} \bar{u}_{n, 0}(t)+Q_{h} \partial \bar{u}_{n, 0}(t)\right)+\left(R_{h}-P_{h}\right) A \bar{u}_{n, 0}(t) \\
R_{h} \bar{u}_{n, 0}(0) & =R_{h} u\left(t_{n}\right) .
\end{aligned}
$$

Then, subtracting (35) from (32),

$$
\begin{aligned}
\bar{U}_{h, n, 0}^{\prime}(t)-R_{h} \bar{u}_{n, 0}^{\prime}(t) & =A_{h, 0}\left(\bar{U}_{h, n, 0}(t)-R_{h} \bar{u}_{n, 0}(t)\right)+\left(P_{h}-R_{h}\right) A \bar{u}_{n, 0}(t) \\
\bar{U}_{h, n, 0}(0)-R_{h} \bar{u}_{n, 0}(0) & =0
\end{aligned}
$$

Solving this problem exactly,

$$
\bar{U}_{h, n, 0}(k)-R_{h} \bar{u}_{n, 0}(k)=\int_{0}^{k} e^{(k-\tau) A_{h, 0}}\left(P_{h}-R_{h}\right) A \bar{u}_{n, 0}(\tau) d \tau,
$$

which is $O\left(k \varepsilon_{h}\right)$ according to (22) and (26) in hypothesis (H3). This can be applied because $A \bar{u}_{n, 0}(\tau) \in Z$ due to (34) and Lemma 5 .

In a similar way,

$$
\begin{aligned}
R_{h} f_{n, j}^{\prime}(t)+L_{h} Q_{h} \partial f_{n, j}^{\prime}(t) & =R_{h} A f_{n, j}(t)+L_{h} Q_{h} \partial f_{n, j}^{\prime}(t) \\
& =L_{h} A f_{n, j}(t)+\left(R_{h}-L_{h}\right) A f_{n, j}(t)+L_{h} Q_{h} \partial A f_{n, j}(t) \\
& =A_{h}\left(R_{h} f_{n, j}(t)+Q_{h} \partial f_{n, j}(t)\right)+\left(R_{h}-P_{h}\right) A f_{n, j}(t), \\
R_{h} f_{n, j}(0) & =R_{h} f\left(t_{n}+c_{j} k\right),
\end{aligned}
$$


and, subtracting from (33),

$$
\begin{aligned}
\bar{F}_{h, n, j}^{\prime}(t)-R_{h} f_{n, j}^{\prime}(t) & =A_{h, 0}\left(\bar{F}_{h, n, j}(t)-R_{h} f_{n, j}(t)\right)+\left(P_{h}-R_{h}\right) A f_{n, j}(t), \\
\bar{F}_{h, n, j}(0)-R_{h} f_{n, j}(0) & =R_{h} f\left(t_{n}+c_{j} k\right)-R_{h} f\left(t_{n}+c_{j} k\right)=0 .
\end{aligned}
$$

Solving this problem exactly,

$$
\bar{F}_{h, n, j}(k)-R_{h} f_{n, j}(k)=\int_{0}^{k} e^{(k-\tau) A_{h, 0}}\left(P_{h}-R_{h}\right) A f_{n, j}(\tau) d \tau,
$$

which is $O\left(k \varepsilon_{h}\right)$ again through the same argument as above. (We take now into account that $A f_{n, j}(\tau) \in Z$ due to (34) and Lemma 6.)

Therefore, we deduce that

$$
R_{h} \bar{u}_{n+1}-\bar{U}_{h, n+1}=R_{h} \hat{\bar{u}}_{n, 0}-\hat{\bar{U}}_{h, n, 0}+k \sum_{j=1}^{s} b_{j}\left(R_{h} \hat{f}_{n, j}-\hat{\bar{F}}_{h, n, j}\right)=O\left(k \varepsilon_{h}\right) .
$$

\subsubsection{Global errors}

We now study the global errors at $t=t_{n}$, which are given by

$$
e_{h, n}=P_{h} u\left(t_{n}\right)-U_{h, n} .
$$

Theorem 12. Under the same assumptions of Theorem 11,

$$
\mathrm{e}_{h, n}=O\left(\gamma_{h} k^{p}+\varepsilon_{h}\right)
$$

where $\gamma_{h}$ and $\varepsilon_{h}$ are those in (24)-(26) and the constants in Landau notation are independent of $k$ and $h$.

Proof. Because of its definition, $e_{h, n+1}$ can be decomposed as

$$
\begin{aligned}
e_{h, n+1} & =P_{h} u\left(t_{n+1}\right)-U_{h, n+1} \\
& =\left(P_{h} u\left(t_{n+1}\right)-R_{h} u\left(t_{n+1}\right)\right)+R_{h} u\left(t_{n+1}\right)-U_{h, n+1} \\
& =O\left(\varepsilon_{h}\right)+R_{h} u\left(t_{n+1}\right)-U_{h, n+1},
\end{aligned}
$$

where (26) has been used. Besides,

$$
\begin{aligned}
R_{h} u\left(t_{n+1}\right)-U_{h, n+1} & =R_{h} u\left(t_{n+1}\right)-\bar{U}_{h, n+1}+\bar{U}_{h, n+1}-U_{h, n+1} \\
& =\rho_{h, n+1}+\bar{U}_{h, n+1}-U_{h, n+1} .
\end{aligned}
$$

We now study $\bar{U}_{h, n+1}-U_{h, n+1}$. Subtracting and considering the previous subsection,

$$
\bar{U}_{h, n+1}-U_{h, n+1}=\hat{\bar{U}}_{h, n, 0}-\hat{U}_{h, n, 0}+k \sum_{j=1}^{s} b_{j}\left(\hat{\bar{F}}_{h, n, j}-\hat{F}_{h, n, j}\right) .
$$


Due to (32) and (28), $\hat{\bar{U}}_{h, n, 0}-\hat{U}_{h, n, 0}$ is the value at $t=k$ of the solution of

$$
\begin{aligned}
\bar{U}_{h, n, 0}^{\prime}(t)-U_{h, n, 0}^{\prime}(t) & =A_{h, 0}\left(\bar{U}_{h, n, 0}(t)-U_{h, n, 0}(t)\right) \\
\bar{U}_{h, n, 0}(0)-U_{h, n, 0}(0) & =R_{h} u\left(t_{n}\right)-U_{h, n}
\end{aligned}
$$

and, therefore, solving exactly,

$$
\hat{\bar{U}}_{h, n, 0}-\hat{U}_{h, n, 0}=\bar{U}_{h, n, 0}(k)-U_{h, n, 0}(k)=e^{k A_{h, 0}}\left(R_{h} u\left(t_{n}\right)-U_{h, n}\right) .
$$

Similarly, from (29) and (33), $\hat{\bar{F}}_{h, n, j}-\hat{F}_{h, n, j}$ is the value at $t=\left(1-c_{j}\right) k$ of the solution of

$$
\begin{aligned}
\bar{F}_{h, n, j}^{\prime}(t)-F_{h, n, j}^{\prime}(t) & =A_{h, 0}\left(\bar{F}_{h, n, j}(t)-F_{h, n, j}(t)\right), \\
\bar{F}_{h, n, j}(0)-F_{h, n, j}(0) & =\left(R_{h}-P_{h}\right) f\left(t_{n}+c_{j} k\right) .
\end{aligned}
$$

Solving this problem exactly,

$\hat{\bar{F}}_{h, n, j}-\hat{F}_{h, n, j}=\bar{F}_{h, n, j}\left(\left(1-c_{j}\right) k\right)-F_{h, n, j}\left(\left(1-c_{j}\right) k\right)=e^{\left(1-c_{j}\right) k A_{h, 0}}\left(R_{h}-P_{h}\right) f\left(t_{n}+c_{j} k\right)$.

Finally, we deduce from this, Theorem 11 and (26), the following recursion formula

$$
\begin{aligned}
& R_{h} u\left(t_{n+1}\right)-U_{h, n+1} \\
& =\rho_{h, n+1}+\bar{U}_{h, n+1}-U_{h, n+1} \\
& =\rho_{h, n+1}+\hat{\bar{U}}_{h, n, 0}-\hat{U}_{h, n, 0}+k \sum_{j=1}^{s} b_{j}\left(\hat{\bar{F}}_{h, n, j}-\hat{F}_{h, n, j}\right) \\
& =\rho_{h, n+1}+e^{k A_{h, 0}}\left(R_{h} u\left(t_{n}\right)-U_{h, n}\right)+k \sum_{j=1}^{s} b_{j} e^{\left(1-c_{j}\right) k A_{h, 0}}\left(R_{h}-P_{h}\right) f\left(t_{n}+c_{j} k\right) \\
& =e^{k A_{h, 0}}\left(R_{h} u\left(t_{n}\right)-U_{h, n}\right)+O\left(\gamma_{h} k^{p+1}\right)+O\left(k \varepsilon_{h}\right) .
\end{aligned}
$$

This implies that

$$
R_{h} u\left(t_{n}\right)-U_{h, n}=e^{t_{n} A_{h, 0}}\left(R_{h} u(0)-U_{h, 0}\right)+O\left(\gamma_{h} k^{p}+\epsilon_{h}\right),
$$

which, together with (30) and (22), proves the theorem.

\section{$5 \quad$ Examples and numerical results}

In this section, we corroborate the results of previous sections by integrating parabolic problems with homogeneous and non-homogeneous Dirichlet boundary conditions. (We remark that it is possible to consider other boundary conditions with slight modifications.) We will use different time Lawson integrators and different spatial discretizations. 


\subsection{Parabolic problem formulation}

Our main reference for this section is [18]. The references $[8,9,20,21,22]$ are also of interest.

Let $\Omega$ be a bounded domain in $\mathbb{R}^{d}(d=1,2,3)$ with a Lipschitz continuous boundary $\partial \Omega$. We consider the second order linear operator

$$
A w:=\sum_{i, j=1}^{d} D_{i}\left(a_{i j} D_{j} w\right)-\sum_{i=1}^{d}\left(D_{i}\left(b_{i} w\right)+c_{i} D_{i} w\right)-a_{0} w
$$

where $D_{i}=\frac{\partial}{\partial x_{i}}$. We assume that the coefficients $a_{i j}(\mathbf{x}), b_{i}(\mathbf{x}), c_{i}(\mathbf{x})$ and $a_{0}(\mathbf{x})$ are real smooth functions on the domain $\bar{\Omega}$ and $-A$ is elliptic, i.e. there exists a constant $\alpha_{0}>0$ such that

$$
\sum_{i, j=1}^{d} a_{i j}(\mathbf{x}) \xi_{i} \xi_{j} \geq \alpha_{0}|\xi|^{2}
$$

for all $\xi \in \mathbb{R}^{d}$ and almost every $\mathbf{x} \in \Omega$.

From the operator $A$, we derive the bilinear form

$$
a(w, v):=\int_{\Omega}\left[\sum_{i, j=1}^{d} a_{i j} \frac{\partial w}{\partial x_{j}} \frac{\partial v}{\partial x_{i}}-\sum_{i=1}^{d}\left(b_{i} w D_{i} v-c_{i} v D_{i} w\right)+a_{0} w v\right],
$$

for $v, w \in V$, which is a suitable subspace of functions defined over $\Omega$.

In the case of a Dirichlet problem, we take $V=H_{0}^{1}(\Omega)$. Then, the bilinear form $a(\cdot, \cdot)$ is well defined and continuous in $V \times V$, i.e.

$$
|a(u, v)| \leq C|| u\left\|_{V}\right\| v \|_{V}, \quad u, v \in V .
$$

Moreover, under suitable conditions, $a(\cdot, \cdot)$ is coercive (see [18], p.164), i.e.

$$
a(u, u) \geq \alpha\|u\|_{V}^{2},
$$

and we deduce that the variational problem "find $u \in V$ such that

$$
a(u, v)=(f, v), \quad v \in V,
$$

is uniquely solvable for $f \in L^{2}(\Omega)$. Moreover, a smooth solution of (36) is also the solution of the homogeneous Dirichlet elliptic problem

$$
\begin{aligned}
-A u & =f, \\
\left.u\right|_{\partial \Omega} & =0 .
\end{aligned}
$$

We are mainly interested in non-homogeneous boundary problems. Assuming that the boundary datum $g$ belongs to $H^{1 / 2}(\partial \Omega), g$ may be extended to the whole $\Omega$ to a function $\widetilde{g} \in H^{1}(\Omega)$ and the variational problem is 
"find $u \in V$ such that

$$
a(u+\widetilde{g}, v)=(f, v), \quad v \in V .
$$

We take $X=L^{2}(\Omega), Y=H^{3 / 2}(\partial \Omega)$ and we denote $D(A)=H^{2}(\Omega) \subset X$. Consider the operator acting on $D(A)$,

$$
\partial u=\left.u\right|_{\partial \Omega}
$$

On the other hand, we consider the operator $A_{0}=\left.A\right|_{\operatorname{ker}(\partial)}$. Then, $D\left(A_{0}\right)=H^{2}(\Omega) \cap$ $H_{0}^{1}(\Omega)$ and $A_{0}$ is the infinitesimal generator of an analytic semigroup $\left\{e^{t A_{0}}\right\}$ in $X$. Therefore, with the notation above, the IBVP

$$
\begin{array}{ll}
u_{t}=A u+f, & \text { on } \Omega \times[0, T], \\
\left.u\right|_{t=0}=u_{0}, & \text { on } \Omega, \\
u=g, & \text { on } \partial \Omega \times[0, T],
\end{array}
$$

can be fitted into the theory of abstract IBVPs developed in $[1,5,16]$ and it can be written as (1).

\subsection{Example 1. Galerkin finite element methods}

Finite elements are used for the semidiscretization of the weak formulation of (38),

"find $u \in L^{2}(0, T ; V) \cap C\left([0, T], L^{2}(\Omega)\right)$ such that

$$
\frac{d}{d t}(u(t)+\widetilde{g}(t), v)+a(u(t)+\widetilde{g}(t), v)=(f, v), \quad v \in V . "
$$

where $u(0)+\widetilde{g}(0)=u_{0}, \widetilde{g}(t)$ is a suitable extension of the boundary datum $g(t)$ in whole $\Omega$ and $f \in L^{2}(\Omega \times(0, T))$. Our approach is closely based on Remark 6.2.2 in $[18]$.

We suppose that $\mathcal{T}_{h}$ is a partition of $\Omega_{h}=\bigcup_{T \in \mathcal{T}_{h}} T$, a suitable subdomain of $\bar{\Omega}$. Moreover, $X=L^{2}(\Omega)$ and $\left\{V_{h}\right\}_{h>0} \subset X$ is a family of finite dimensional spaces with the inherited norm which is made up of finite elements. We suppose that the partition $\Omega_{h}=\bigcup_{T \in \mathcal{T}_{h}} T$ and the finite elements satisfy the assumptions in Theorem 6.2.1 in [18].

Let us take $X_{h}=V_{h}$. The elements of $X_{h}$ are functions which are defined as piecewise polynomial interpolants of their values in the nodes associated to the partition $\mathcal{T}_{h}$. Some of these nodes are on the boundary and we denote $X_{h, b} \subset X_{h}$ to the subspace formed by the polynomial functions which vanish on the internal nodes and $X_{h, 0} \subset X_{h}$ to the subspace formed by the piecewise polynomial functions which vanish on the boundary nodes. Then, we can write $X_{h}=X_{h, 0} \oplus X_{h, b}$. We denote by $Q_{h} \partial u \in X_{h, b}$ to the piecewise interpolating polynomial of the boundary values of $u \in D(A)$. Therefore, in (24) inside hypothesis (H2), the factor $\gamma_{h}$ depends on the approximation of the domain $\Omega$ and on the boundary condition (see for example section 4.4 in [20]). In particular, when the boundary conditions are Dirichlet and the norm is the $L^{2}$-norm, $\gamma_{h}=O(1)$. 


\begin{tabular}{|c|c|c|c|c|c|}
\hline$h$ & $k=0.1$ & $k=0.05$ & $k=0.025$ & $k=0.0125$ & $k=0.00625$ \\
\hline 0.01 & $2.0739 \times 10^{2}$ & $1.0366 \times 10^{2}$ & $5.1801 \times 10^{1}$ & $2.5871 \times 10^{1}$ & $1.2906 \times 10^{1}$ \\
\hline 0.005 & $5.8673 \times 10^{2}$ & $2.9334 \times 10^{2}$ & $1.4665 \times 10^{2}$ & $7.3303 \times 10^{1}$ & $3.6630 \times 10^{1}$ \\
\hline 0.0025 & $1.6596 \times 10^{3}$ & $8.2980 \times 10^{2}$ & $4.1488 \times 10^{2}$ & $2.0743 \times 10^{2}$ & $1.0370 \times 10^{2}$ \\
\hline
\end{tabular}

Table 1: $L^{2}$-global error when $h$ decreases when integrating problem (40) through (21), with quadratic finite elements in space and trapezoidal Lawson rule in time

As the projection operator $L_{h}: X \rightarrow X_{h, 0}$, we take the orthogonal projection which is defined by

$$
\left(L_{h} u, \chi\right)=(u, \chi), \quad u \in X=L^{2}(\Omega), \chi \in X_{h, 0},
$$

which satisfies (23) inside hypothesis (H2).

The operators $A_{h}: X_{h} \rightarrow X_{h, 0}$ are defined through the relation

$$
\left(A_{h} u_{h}, \chi\right)=-a\left(u_{h}, \chi\right), \quad u_{h} \in X_{h}, \quad \chi \in X_{h, 0},
$$

and $A_{h, 0}$ is the restriction of $A_{h}$ to $X_{h, 0}$. Then, the operators $A_{h, 0}$ are invertible and generate analytic semigroups on $X_{h}$ satisfying (H1) (see [9], section 6 and 7). The Ritz (or elliptic) projection is defined by

$$
\left(A_{h}\left(R_{h} u+Q_{h} \partial u\right), \chi\right)=(A u, \chi)=\left(L_{h} A u, \chi\right),
$$

for $u \in D(A)$ and $\chi \in X_{h, 0}$.

We assume that the solution $u$ of problem (38) is in the space $H^{s}(\Omega)$, for certain $s>0$. Since $D\left(A^{r}\right)=H^{2 r}(\Omega)$, for any $r>0,(34)$ is satisfied for $Z=H^{2 r}(\Omega)$ whenever $u(t) \in H^{2(p+1+r)}(\Omega)$ and $f(t) \in H^{2(p+r)}(\Omega)$. Moreover, with suitable hypotheses on the finite elements being used (see e.g. Remark 6.2.2 in [18]), we can obtain (26) with

$$
\varepsilon_{h}=O\left(h^{l}\right), \quad \text { for } l=\min (m, r-1),
$$

where we suppose that the basis functions of $X_{h}$ are included in the space of piecewisepolynomials of degree less than or equal to $m$. The estimate on the global error which is obtained then in Theorem 12 is

$$
O\left(k^{p}+h^{l}\right)
$$

with $l=\min (m, r-1)$.

In the first place, we have considered the following Dirichlet problem with nonvanishing boundary conditions:

$$
\begin{aligned}
u_{t}(x, t) & =u_{x x}(x, t)-2 e^{x-t}, \quad x \in[0,1], \quad t>0 \\
u(0, t) & =e^{-t} \\
u(1, t) & =e^{1-t} \\
u(x, 0) & =e^{x} .
\end{aligned}
$$




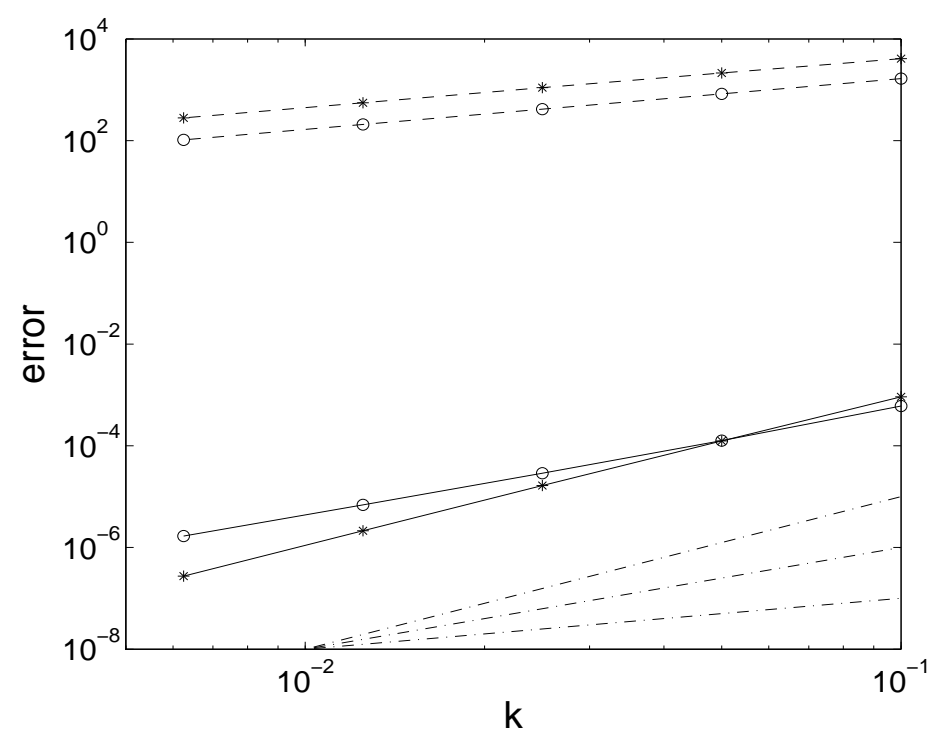

Figure 1: Local error $\left(^{*}\right)$ and global error (o) without avoiding (discont.) and avoiding (cont.) order reduction when integrating problem (40) with trapezoidal Lawson rule, (dash-dotted lines: slopes 1,2 and 3 ), $h=2.5 \times 10^{-3}, k=0.1,0.05, \ldots$

\begin{tabular}{|c|c|c|c|c|}
\hline Local error order without avoiding O. R. & 0.93 & 0.96 & 0.98 & 0.99 \\
\hline Local error order avoiding O. R. & 2.88 & 2.92 & 2.95 & 2.96 \\
\hline Global error order without avoiding O. R. & 1.00 & 1.00 & 1.00 & 1.00 \\
\hline Global error order avoiding O. R. & 2.26 & 2.13 & 2.06 & 2.03 \\
\hline
\end{tabular}

Table 2: Order corresponding to the integration of problem (40), with quadratic finite elements in space and trapezoidal Lawson rule in time, $h=2.5 \times 10^{-3}, k=0.1,0.05, \ldots$

whose exact solution is $u(x, t)=e^{x-t}$. For the space discretization, we have used quadratic finite element methods, for which $m=2$ in (39). As for the time integration, we have chosen the Lawson method which is based on the quadrature rule approximation corresponding to $c_{1}=0, c_{2}=1, b_{1}=b_{2}=1 / 2$, which is the trapezoidal rule. This method, when applied to (9), is well-known to have order $p=2$. We have firstly integrated the problem without avoiding order reduction, by solving (21) directly. Then, we have integrated it by using the suggested formula (31). We have measured the error when using the method of lines without avoiding order reduction and with the technique suggested here. More precisely, we measure the $L^{2}$-norm of the difference between the piecewise quadratic interpolants of the exact solution and the obtained numerical solution. Figure 1 shows error just after a timestepsize (local error, ${ }^{*}$ ) and at a final time $t=1$ (global error, o) when using as space grid $h=2.5 \times 10^{-3}$ and timestepsizes $k=0.1,0.05, \ldots$ We can observe that the errors, without avoiding order reduction, are completely unacceptable. In fact, the errors are bigger and bigger when- 


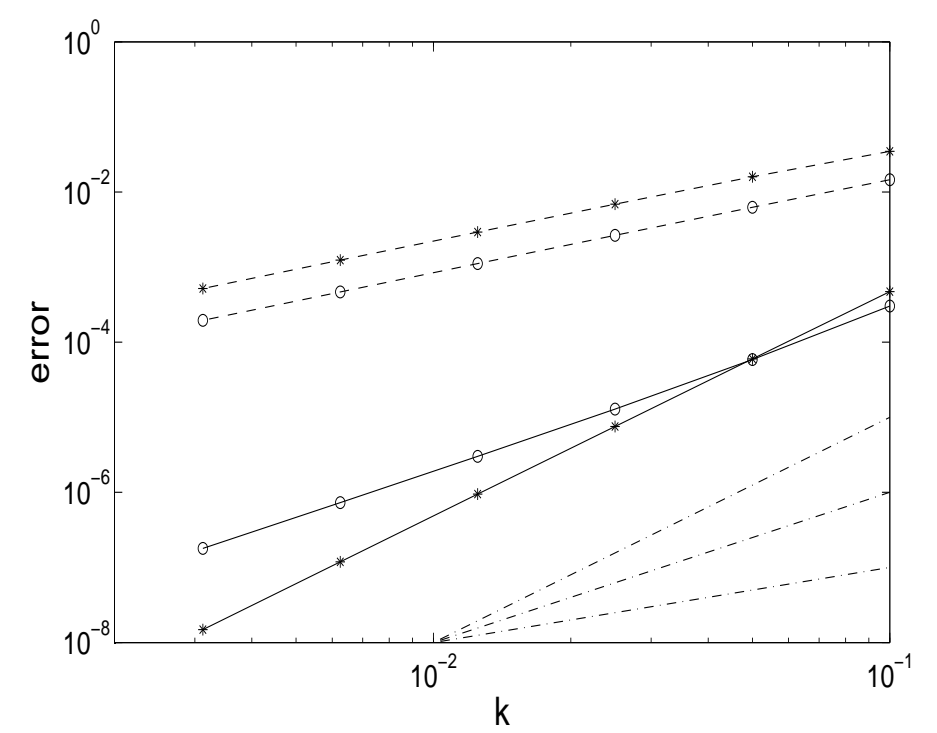

Figure 2: Local error $(*)$ and global error (o) without avoiding (discont.) and avoiding (cont.) order reduction when integrating problem (41) with trapezoidal Lawson rule (dash-dotted lines: slopes 1, 2 and 3), $h=2.5 \times 10^{-3}, k=0.1,0.05, \ldots$

\begin{tabular}{|c|c|c|c|c|c|}
\hline Local error order without avoiding O. R. & 1.13 & 1.21 & 1.23 & 1.24 & 1.25 \\
\hline Local error order avoiding O. R. & 2.98 & 2.99 & 2.99 & 3.00 & 3.00 \\
\hline Global error order without avoiding O. R. & 1.22 & 1.24 & 1.25 & 1.25 & 1.26 \\
\hline Global error order avoiding O. R. & 2.37 & 2.19 & 2.09 & 2.05 & 2.02 \\
\hline
\end{tabular}

Table 3: Order corresponding to the integration of problem (41), with quadratic finite elements in space and trapezoidal Lawson rule in time, $h=2.5 \times 10^{-3}, k=0.1,0.05, \ldots$.

ever $h$ diminishes. (See Table 1.) However, with the technique suggested in this paper, we achieve to integrate the problem in an accurate way and, what's more, avoiding completely order reduction. That can be observed in the slope of lines of Figure 1 and also in Table 2, where the precise values of the order (estimated from consecutive values of the error) are written. This corroborates Theorems 11 and 12 since order 3 for the local error and order 2 for the global one is observed.

In the second place, we have considered the vanishing boundary value problem

$$
\begin{aligned}
u_{t}(x, t) & =u_{x x}(x, t)+\left(x^{2}-x+2\right) e^{-t}, \quad x \in[0,1], \quad t>0, \\
u(0, t) & =u(1, t)=0,
\end{aligned}
$$

whose exact solution is $u(x, t)=x(1-x) e^{-t}$. We have discretized it in the same way than the previous problem. We notice in Figure 2 that, when avoiding order reduction, not only the slope of the lines increases, but also the size of the errors is much smaller even for the biggest values of $k$. The precise values of the order are written in Table 3. 
The second and fourth line of the table corroborate Theorems 11 and 12 because order 3 for the local error and order 2 for the global one are observed when order reduction is avoided. This means a significant improvement in contrast with the order 1.25 which is observed for the local and global errors without avoiding order reduction, as it was justified in [4].

\subsection{Example 2. Finite-difference schemes}

The finite-difference case can also be analysed under the framework in Section 4.1. Now, we can consider $X=C(\Omega)$ and $Y=C(\partial \Omega)$. Besides, for each parameter $h$, we can consider a grid $\Omega_{h}$ with some interior nodes in the interior of $\Omega$ and some boundary nodes in $\partial \Omega$. Associated to each $\Omega_{h}$, we can think of $\left\{X_{h}\right\}$ as any finite dimensional family of subspaces of $X$ such that there is a one-to-one correspondence between each element of $X_{h}$ and its nodal values in $\Omega_{h}$. The interior nodal values determine the element in $X_{h, 0}$ (for which the nodal values on the boundary vanish) while the boundary ones do the same in $X_{h, b}$ (for which the nodal values in the interior vanish). The corresponding norm in $X_{h}$ will be the discrete $L^{2}$-norm. We will consider the interpolating operator $Q_{h}: Y \rightarrow X_{h, b}$, which takes any $g \in Y$ to the function in $X_{h, b}$ whose nodal values on the boundary of $\Omega_{h}$ coincide with those of $g$. Then, the restriction of the operator $A_{h}: X_{h} \rightarrow X_{h, 0}$ (which approximates $A$ ) to $X_{h, 0}$ will be represented by a certain square matrix, which we will denote as $B_{h, 0}$.

\subsubsection{1-dimensional problem}

We will firstly discretize problem (40) in space with the classical symmetric secondorder FD scheme for the second derivative. For the discretization of the

$$
w_{x x}(x)=f(x), \quad x \in(0,1), \quad w(0)=w_{0}, w(1)=w_{1},
$$

this can be written as

$$
B_{h, 0} W_{h, 0}+E_{h}\left[w_{0} w_{1}\right]^{T}=f_{h, 0},
$$

where $W_{h, 0}$ is the vector which contains the searched interior grid nodal values of $w$, $f_{h, 0}$ is the vector which contains the interior grid nodal values of $f$ and

$$
B_{h, 0}=\frac{1}{h^{2}}\left[\begin{array}{ccccc}
-2 & 1 & 0 & & \\
1 & -2 & 1 & \ddots & \\
0 & 1 & -2 & \ddots & 0 \\
& \ddots & \ddots & \ddots & 1 \\
& & 0 & 1 & -2
\end{array}\right], \quad E_{h}\left[\begin{array}{c}
w_{0} \\
w_{1}
\end{array}\right]=\frac{1}{h^{2}}\left[\begin{array}{c}
w_{0} \\
0 \\
\vdots \\
0 \\
w_{1}
\end{array}\right]
$$

In such a way, using the framework in (25), $R_{h} w$ is represented by $W_{h, 0}, B_{h, 0}$ is the matrix which represents $A_{h, 0}$ and $E_{h}$ is the one which represents $A_{h} Q_{h}, L_{h}$ is just the inside nodal projection and therefore, in this case, $L_{h} Q_{h} \equiv 0$, which implies that $L_{h} \equiv P_{h}$. As it is well known, the eigenvalues of $B_{h, 0}$ are negative, which implies (H1), 


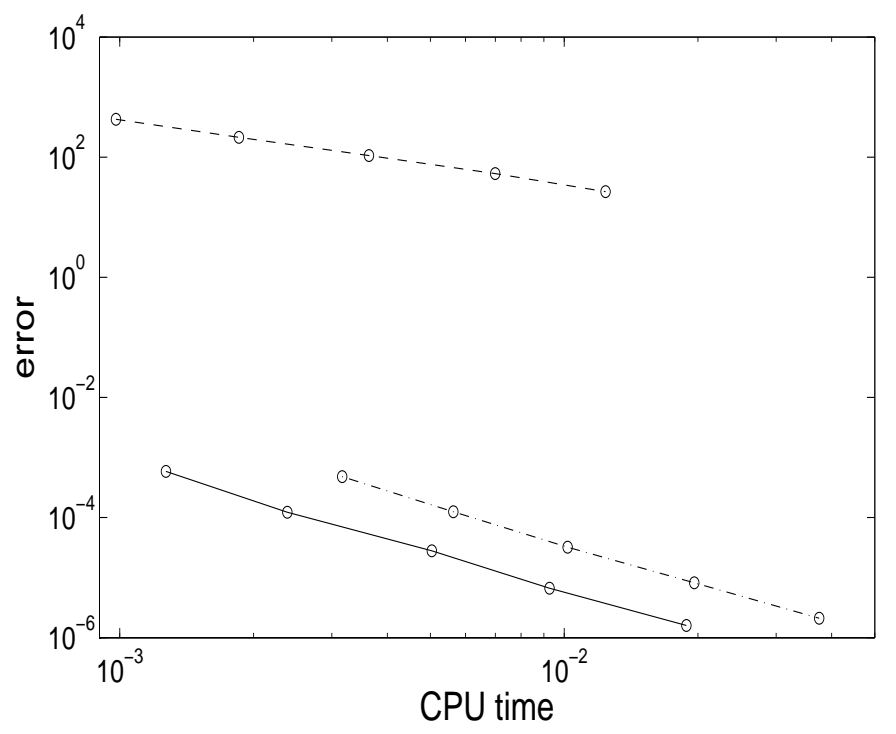

Figure 3: Global error against cpu time when integrating (40) with trapezoidal Lawson quadrature rule without avoiding order reduction (dashed line), avoiding order reduction (continuous line) and with the exponential quadrature rule (44) (dash-dotted line), $h=2.5 \times 10^{-3}, k=0.1,0.05, \ldots$ (Implementation calculating exponential-type matrices once and for all at the very beginning.)

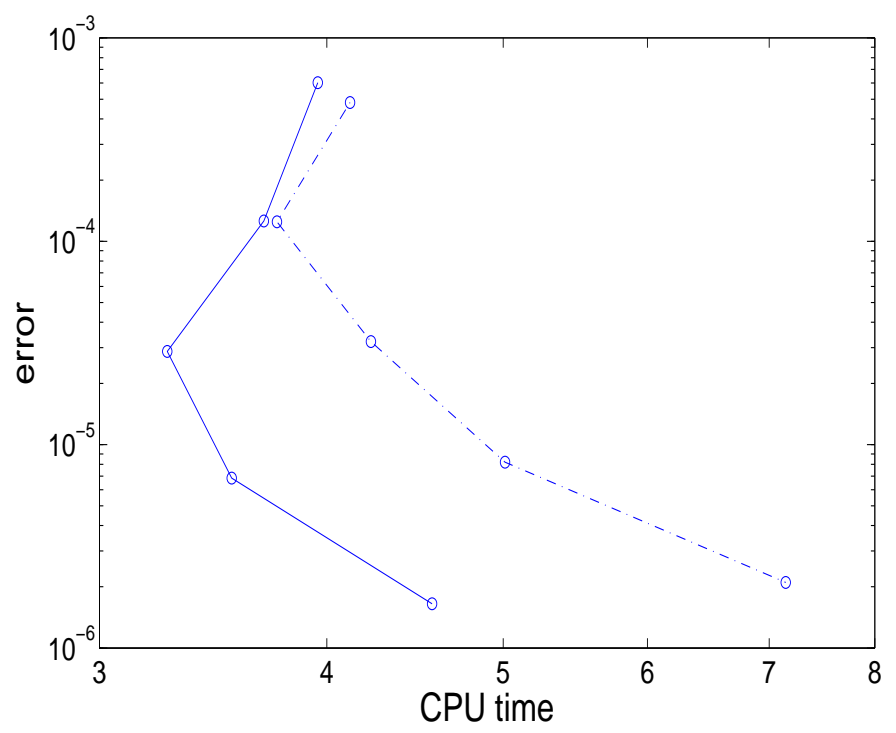

Figure 4: Global error against cpu time when integrating (40) with trapezoidal Lawson quadrature rule avoiding order reduction (continuous line) and with the exponential quadrature rule (44) (dash-dotted line), $h=2.5 \times 10^{-3}, k=0.1,0.05, \ldots$ (Implementation with Krylov techniques.) 
and (23) and (24) in (H2) are clear. On the other hand, (H3) is also well-known to be satisfied with $Z=H^{4}(0,1)$ and $\epsilon_{h}=O\left(h^{2}\|w\|_{H^{4}(0,1)}\right)$.

Notice that, in this particular case, when integrating in time with trapezoidal Lawson rule using the implementation in (31), the following scheme turns up:

$$
\begin{aligned}
U_{h, n+1}= & e^{k A_{h, 0}}\left[U_{h, n}+\frac{k}{2} P_{h} f\left(t_{n}\right)\right]+\frac{k}{2} P_{h} f\left(t_{n+1}\right)+k \varphi_{1}\left(k A_{h, 0}\right) A_{h} Q_{h} \partial\left[u\left(t_{n}\right)+\frac{k}{2} f\left(t_{n}\right)\right] \\
& +k^{2} \varphi_{2}\left(k A_{h, 0}\right) A_{h} Q_{h} \partial\left[A u\left(t_{n}\right)+\frac{k}{2} A f\left(t_{n}\right)\right]+k^{3} \varphi_{3}\left(k A_{h, 0}\right) A_{h} Q_{h} \partial A^{2} u\left(t_{n}\right) .(42)
\end{aligned}
$$

Therefore, the only terms which must be calculated, apart from those which appear when applying trapezoidal Lawson method without avoiding order reduction to a vanishing boundary conditions problem, are, for $j=1,2$,

$$
\frac{k^{j}}{h^{2}} \varphi_{j}\left(k B_{h, 0}\right)\left[\begin{array}{c}
A^{j-1} u\left(0, t_{n}\right)+\frac{k}{2} A^{j-1} f\left(0, t_{n}\right) \\
0 \\
\vdots \\
0 \\
A^{j-1} u\left(1, t_{n}\right)+\frac{k}{2} A^{j-1} f\left(1, t_{n}\right)
\end{array}\right], \quad \frac{k^{3}}{h^{2}} \varphi_{3}\left(k B_{h, 0}\right)\left[\begin{array}{c}
A^{2} u\left(0, t_{n}\right) \\
0 \\
\vdots \\
0 \\
A^{2} u\left(1, t_{n}\right)
\end{array}\right] .
$$

When the stepsize is fixed, as we assume in our analysis, what is just required is then to calculate the first and last column of $\varphi_{j}\left(k B_{h, 0}\right)(j=1,2,3)$ once and for all at the very beginning, and then at each step just the corresponding linear combination of those two columns must be performed. As distinct, all columns of $e^{k B_{h, 0}}$ should be calculated since, in principle, $U_{h, n}$ and $P_{h} f\left(t_{n}\right)$ have no vanishing components. Because of that, the cost of computing (43) is negligible compared with that of calculating the first term in (42).

The global discrete $L^{2}$-errors have been calculated without avoiding and avoiding order reduction. The results against CPU time when integrating till time $t=1$ are shown in Figure 3. The time required to calculate the exponential-type matrices has not been considered since those calculations are performed just at the very beginning, and the relative cost of that part would very much depend on the final time of integration. The values of $k$ and $h$ which have been considered are the same as in Figure 1 and, as the error in space is negligible with respect to that in time, the values for the errors are practically the same as in that figure. It is clear that, for each value of $k$, avoiding order reduction implies a big reduction on the size of the error but a very small increase in computational time. Moreover, although it is not an aim of the paper to recommend any particular method, we have compared the results with the exponential quadrature rule which is based on interpolating $F$ in (11) by a linear polynomial. When integrating (21) with the mentioned rule and the above space discretization, for which $L_{h} Q_{h} \equiv 0$, the scheme is given by

$$
\begin{aligned}
U_{h, n+1}= & e^{k A_{h, 0}} U_{h, n}+k \varphi_{1}\left(k A_{h, 0}\right)\left[A_{h} Q_{h} g\left(t_{n}\right)+P_{h} f\left(t_{n}\right)\right] \\
& \left.+k \varphi_{2}\left(k A_{h, 0}\right)\right)\left[A_{h} Q_{h}\left(g\left(t_{n+1}\right)-g\left(t_{n}\right)\right)+P_{h} f\left(t_{n+1}\right)-P_{h} f\left(t_{n}\right)\right] .
\end{aligned}
$$

This formula has been proved to have stiff order 2 for homogeneous boundary conditions [10] and it happens to show the same order for our non-homogeneous boundary 


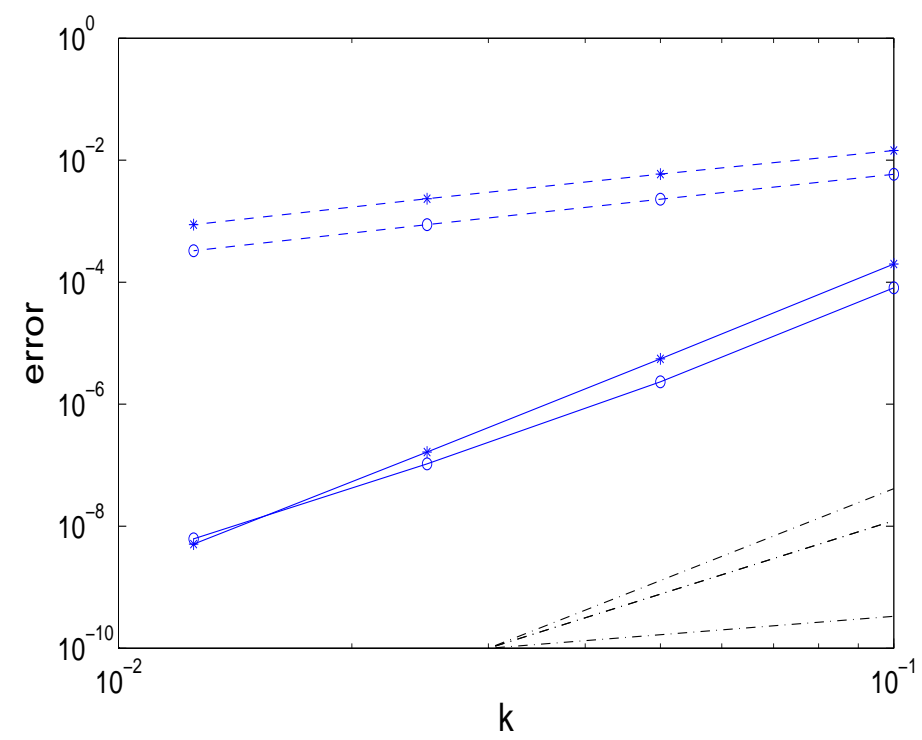

Figure 5: Local error $(*)$ and global error (o) without avoiding (discont.) and avoiding (cont.) order reduction when integrating problem (45) with vanishing boundary conditions with Lawson Simpson rule (dash-dotted lines: slopes 1, 4 and 5), $\mathrm{h}=0.01$, $\mathrm{k}=0.1,0.05, \ldots$

conditions problem. However, as it can also be observed in Figure 3, it is approximately twice more expensive than trapezoidal Lawson rule in this problem. The reason for that is that $\varphi_{1}\left(k B_{h, 0}\right)$ and $\varphi_{2}\left(k B_{h, 0}\right)$ must be calculated over full non-zero vectors. Notice that, if the number of stages with other exponential methods had to be increased to achieve a higher stiff order, the comparison would be even more beneficial for the implementation of Lawson methods as suggested here since the classical order is obtained without increasing the number of stages.

On the other hand, we have also made the numerical comparison between both second-order methods calculating the actions of the exponential-type matrices over vectors through Krylov techniques. In such a way, it is not necessary to calculate the exponential-type matrices at the very beginning, and the procedure could also be applied with variable stepsizes. For that, we have used the subroutines in [13] with the default values for the parameters in them. Figure 4 shows the results that we have obtained. At least for that problem, for the smallest values of $k$, the technique described here to implement the trapezoidal Lawson method is a bit cheaper than the mentioned exponential quadrature rule.

\subsubsection{2-dimensional problems}

In this subsection we have considered two particular problems in two dimensions. One corresponds to vanishing boundary conditions and another one to non-vanishing boundary conditions, for which the standard Lawson method gives completely unacceptable 
results. The problem is

$$
\begin{aligned}
u_{t}(t, x, y) & =u_{x x}(t, x, y)+u_{y y}(t, x, y)+f(t, x, y), \quad t>0, \quad(x, y) \in \Omega=(0,1) \times(0,1), \\
u(t, x, y) & =g(t, x, y), \quad(x, y) \in \partial \Omega,
\end{aligned}
$$

with functions $f(t, x, y)$ and $g(t, x, y)$ such that the exact solutions are

$$
u(t, x, y)=x(1-x) y(1-y) e^{x+y-t} \text { and } u(t, x, y)=e^{x+y-t} .
$$

For the space discretization of the Laplacian, we have considered the well-known fourthorder nine-point formula [19] while for the time integration we have used Lawson method based on Simpson's quadrature rule, which is also of fourth order for problem (9). More precisely, the scheme for the discretization of the Laplacian in a square,

$$
w_{x x}(x, y)+w_{y y}(x, y)=f(x, y), \quad \partial w=g \text { in } \partial \Omega,
$$

can be written (in terms of the searched nodal values in the interior $W_{h, 0}$, the given values of $g$ on the boundary $w_{h, b}$ and the values of $f$ in the interior and boundary grid nodes $f_{h, 0}$ and $\left.f_{h, b}\right)$ as

$$
C_{h} W_{h, 0}+D_{h} w_{h, b}=M_{h} f_{h, 0}+N_{h} f_{h, b},
$$

where $C_{h}$ and $M_{h}$ are the tridiagonal block matrices

$$
\begin{aligned}
& C_{h}=\frac{1}{h^{2}}\left[\begin{array}{ccccc}
-\frac{10}{3} I+\frac{2}{3} J & \frac{2}{3} I+\frac{1}{6} J & 0 & \cdots & 0 \\
\frac{2}{3} I+\frac{1}{6} J & -\frac{10}{3} I+\frac{2}{3} J & \ddots & & \vdots \\
0 & \ddots & \ddots & \ddots & 0 \\
\vdots & & \ddots & \ddots & \frac{2}{3} I+\frac{1}{6} J \\
0 & \ldots & 0 & \frac{2}{3} I+\frac{1}{6} J & -\frac{10}{3} I+\frac{2}{3} J
\end{array}\right], \\
& M_{h}=\frac{1}{12}\left[\begin{array}{ccccc}
8 I+J & I & 0 & \cdots & 0 \\
I & 8 I+J & \ddots & & \vdots \\
0 & \ddots & \ddots & \ddots & 0 \\
\vdots & & \ddots & \ddots & I \\
0 & \ldots & 0 & I & 8 I+J
\end{array}\right]
\end{aligned}
$$

with

$$
J=\left[\begin{array}{ccccc}
0 & 1 & 0 & \ldots & 0 \\
1 & 0 & 1 & & \vdots \\
0 & \ddots & \ddots & \ddots & \vdots \\
\vdots & & \ddots & \ddots & 1 \\
0 & \ldots & 0 & 1 & 0
\end{array}\right]
$$

and $D_{h}, N_{h}$ correspond respectively to the associated block-matrices in $C_{h}$ and $M_{h}$ acting on the boundary. This can be written as

$$
M_{h}^{-1} C_{h} W_{h, 0}+M_{h}^{-1} D_{h} w_{h, b}=f_{h, 0}+M_{h}^{-1} N_{h} f_{h, b},
$$




\begin{tabular}{|c|c|c|c|}
\hline Local error order without avoiding O. R. & 1.29 & 1.34 & 1.41 \\
\hline Local error order avoiding O. R. & 5.17 & 5.07 & 5.01 \\
\hline Global error order without avoiding O. R. & 1.36 & 1.38 & 1.43 \\
\hline Global error order avoiding O. R. & 5.11 & 4.47 & 4.09 \\
\hline
\end{tabular}

Table 4: Order corresponding to the integration of problem (45) with vanishing boundary conditions, with nine-point formula in space and Simpson Lawson rule in time, $h=0.01, k=0.1,0.05, \ldots$

which resembles (25), where $B_{h, 0}=M_{h}^{-1} C_{h}$ represents the discretization $A_{h, 0}$ and the operator $L_{h}$, when applied to the function $f$ is represented by $f_{h, 0}+M_{h}^{-1} N_{h} f_{h, b}$. In such a way, $P_{h} f=L_{h} f-L_{h} Q_{h} f$ is represented by the interior nodal values of $f\left(f_{h, 0}\right)$.

From the form of the matrices $C_{h}$ and $M_{h}$ (47)-(48), it is easy to see that both matrices are symmetric and commute. Because of this, they diagonallize in the same base of eigenvectors. Applying Gerschgorin theorem, the eigenvalues of $C_{h}$ are negative and those of $M_{h}$ are positive. Therefore, the eigenvalues of $B_{h, 0}=M_{h}^{-1} C_{h}$ are negative, which implies (H1) with $M=1$. On the other hand, (24) in (H2) is clear for $\gamma_{h}=1$. Besides, because of the fact that the eigenvalues of $M_{h}$ are in $\left(\frac{1}{3}, 1\right)$ by Gerschgorin theorem, the Euclidean norm of $M_{h}^{-1}$ (which is symmetric) is bounded by 3 . If we add to that the boundedness of the coefficients of $N_{h},(23)$ in (H2) follows. Finally, from $[19]$

$$
C_{h} w_{h, 0}+D_{h} w_{h, b}=M_{h} f_{h, 0}+N_{h} f_{h, b}+O\left(h^{4}\|w\|_{H^{6}(\Omega)}\right),
$$

where $w_{h, 0}$ is the vector which represents $P_{h} w$ and has the interior nodal values of $w$. Subtracting from (46) and bounding in the corresponding norm,

$$
\left\|w_{h, 0}-W_{h, 0}\right\|=O\left(h^{4}\|w\|_{H^{6}(\Omega)}\left\|C_{h}^{-1}\right\|\right) .
$$

(Notice that $h^{2} C_{h}$ can be written as the Kronecker product $\frac{1}{6}(4 I+J) \otimes(4 I+J)-6 I$ and, as the eigenvalues of $J$ are $\{2 \cos (2 \pi j / N)\}_{j=1, \ldots, N-1}$ with $N$ such that $N h$ equals the side of the square, the eigenvalues of $h^{2} C_{h}$ are $\left\{\frac{1}{6}(4+2 \cos (2 \pi j / N))(4+2 \cos (2 \pi l / N))-\right.$ $6\}_{j, l=1, \ldots, N-1}$. From this it is clear that the smallest in modulus of the eigenvalues is $O\left(h^{2}\right)$ and therefore $C_{h}^{-1}$ is bounded independently of $h$.) As $W_{h, 0}$ is the vector which represents $R_{h} w,(26)$ in (H3) follows with $Z=H^{6}(\Omega)$ and $\varepsilon_{h}=O\left(h^{4}\right)$.

Again the local and global discrete $L^{2}$-errors have been calculated for the vanishing boundary conditions problem without avoiding and avoiding order reduction. The results when using a uniform grid space with $h=0.01$ and time stepsizes $k=0.1,0.05, \ldots$ are shown in Figure 5. (The errors in the space discretization are then negligible against time discretization errors.) Again the advantages of using the technique suggested in the paper are obvious in terms of order and in terms of the magnitude of the errors. Moreover, the biggest the classical order the biggest the benefits of avoiding order reduction. Table 4 confirms more precisely the results on the recovery of the classical order, where now the order is estimated from the consecutive values of the errors shown in Figure 5. 


\begin{tabular}{|c|c|c|c|}
\hline Local error order avoiding O. R. & 4.67 & 4.73 & 4.86 \\
\hline Global error order avoiding O. R. & 4.27 & 4.10 & 4.09 \\
\hline
\end{tabular}

Table 5: Order corresponding to the integration of problem (45) with non-vanishing boundary conditions, with nine-point formula in space and Simpson Lawson rule in time, $h=0.01, k=0.1,0.05, \ldots$

As for the problem with non-vanishing boundary conditions, we notice that it is not so easy to reduce it to a problem with vanishing boundary conditions for which there is no order reduction (see the first paragraph of Section 4). The technique suggested in this paper offers an easy and cheap way to tackle the problem which, at the same time, has no order reduction. Table 5 confirms this excellent result, showing again the orders estimated from consecutive errors for the same values of $h$ and $k$ as before.

\section{Acknowledgements}

This research has been supported by Ministerio de Ciencia e Innovación project MTM201566837-P.

\section{References}

[1] I. Alonso-Mallo, Rational methods with optimal order of convergence for partial differential equations, Appl. Num. Math. 35 (1999), 117-131.

[2] I. Alonso-Mallo, Runge-Kutta methods without order reduction for linear initial boundary value problems, Numerische Mathematik 91 (2002), 577-603.

[3] I. Alonso-Mallo and B. Cano, Spectral/Rosenbrock discretizations without order reduction for linear parabolic problems, Appl. Num. Math. 47 (2002), pp. 247-268.

[4] I. Alonso-Mallo, B. Cano and N. Reguera, Analysis of order reduction when integrating linear initial boundary value problems with Lawson methods, submitted for publication.

[5] I. Alonso-Mallo and C. Palencia, On the convolutions operators arising in the study of abstract initial boundary value problems, Proc. Royal Soc. Edinburgh. 126A (1996), 515-539.

[6] P. Brenner, M. Crouzeix and V. Thomée, Single step methods for non-homogeneous linear differential equations in Banach spaces, R.A.I.R.O. An. Num. 16 (1982), 5-26.

[7] M.P. Calvo and C. Palencia, Avoiding the order reduction of Runge-Kutta methods for linear initial boundary value problems, Math. Comput. 71 (2001), 1529-1543. 
[8] P.G. Ciarlet, Basic error Estimates for Elliptic Problems, Vol. II, Part 1, in Handbook of Numerical Analysis, P. G. Ciarlet and J. L. Lions, eds., (North-Holland, Amsterdam, 1991)

[9] H. Fujita and T. Suzuki, Evolution Problems, Vol. II, Part 1, in Handbook of Numerical Analysis, P. G. Ciarlet and J. L. Lions, eds., (North-Holland, Amsterdam, 1991)

[10] M. Hochbruck and A. Ostermann, Explicit exponential Runge-Kutta methods for semilinear parabolic problems, SIAM J. Num. Anal. 43 (2005), 1069-1090.

[11] M. Hochbruck and A. Ostermann, Exponential integrators, Acta Numerica (2010) 209-286.

[12] J. D. Lawson, Generalized Runge-Kutta processes for stable systems with large Lipschitz constants, SIAM J. Numer. Anal. 4 (1967) 372-380.

[13] J. Niesen, and W. M. Wright, Algorithm 919: a Krylov subspace algorithm for evaluating the $\varphi$-functions appearing in exponential integrators, ACM Trans. Math. Software 38, no. 3, Art. 22 (2012).

[14] S. Nörsett, The Numerical Solution of Stiff Systems, Thesis, University of Oslo, Norway, 1969.

[15] C. Palencia, Maximum norm analysis of completely discrete finite element methods for parabolic problems Banach spaces, SIAM J. Numer. Anal. 33 (1993), 1654-1668.

[16] C. Palencia and I. Alonso-Mallo, Abstract initial boundary value problems, Proc. Royal Soc. Edinburgh 124A (1994), 879-908.

[17] A. Pazy, Semigroups of Linear Operators and Applications to Partial Differential Equations, Series: Applied Mathematical Sciences, Vol. 44, Springer, New York, Berlin, Heidelberg, Tokyo, 1983.

[18] A. Quarteroni and A. Valli, Numerical Approximation of Partial Differential Equations, (Springer-verlag, Berlin, 1994)

[19] J. C. Strikwerda, Finite Difference Schemes and Partial Differential Equations, (Wadsworth \& Brooks, United States of America, 1989).

[20] G. Strang and G.J. Fix, An Analysis of the Finite Element Method, (Prentice-Hall, Englewood Cliffs, NJ, 1973).

[21] V. Thomée, Galerkin finite element methods for parabolic problems, in Lecture notes in Math., 1054, (Springer Verlag, Berlin, 1984)

[22] L.B. Wahlbin, Finite element methods for evolution equations, in Advances in Numerical Analysis, Vol. 1, W. Light, ed., (Clarendon Press, Oxford, 1991) 